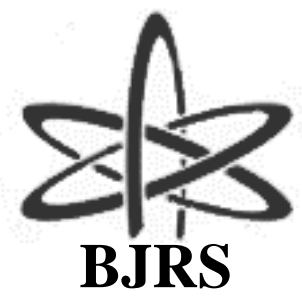

\author{
BRAZILIAN JOURNAL \\ $\mathrm{OF}$ \\ RADIATION SCIENCES \\ 07-03A (2019) 01-09
}

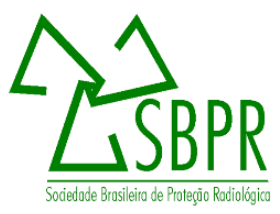

\title{
Amostras grandes analisadas por ativação neutrônica, método $k_{0}$
}

\author{
Sathler $^{a}$ M.M., Salles ${ }^{b}$ P.M.B., OLIVEIRA ${ }^{a}$ H.S., MENEZES ${ }^{a, b}$ M.A.B.C. \\ ${ }^{a}$ Centro de Desenvolvimento da Tecnologia Nuclear (CDTN/CNEN) \\ ${ }^{b}$ Universidade Federal de Minas Gerais, Escola de Engenharia, Departamento de Engenharia Nuclear \\ Av. Presidente Antônio Carlos, 6627, Campus UFMG, CEP 31270-901, Belo Horizonte, Minas Gerais, Brazil \\ menezes@cdtn.br
}

\section{RESUMO}

O Laboratório de Análise por Ativação Neutrônica do Centro de Desenvolvimento da Tecnologia Nuclear, Comissão Nacional de Energia Nuclear, CDTN/CNEN, Belo Horizonte tem desenvolvido atividades analíticas desde o início de operação do reator nuclear de pesquisa TRIGA MARK I IPR-R1 em 1960. Além do método relativo de análise por ativação neutrônica, tem sido aplicado o método $k_{0}$-padronizado, em que os cálculos de concentração dos elementos químicos não se baseiam em padrões dos elementos de interesse e sim no uso de monitores de fluxo de nêutrons, espectrômetro gama absolutamente calibrado, conhecimento de parâmetros espectrais na posição de irradiação do reator nuclear e constantes nucleares compostas que caracterizam os nuclídeos, os chamados fatores $k$. Ao longo do tempo, vários melhoramentos têm sido efetivados nos procedimentos de análise no laboratório, entre eles, o estabelecimento da metodologia para analisar amostras consideradas grandes, de 1 a 5 g, quando o usual em ativação neutrônica é analisar amostra pequena ou puntual com cerca de $200 \mathrm{mg}$. A metodologia consiste em analisar amostras com geometria cilíndrica, aplicando o método $k_{0}$, sem alterar as instalações locais. Neste trabalho são mostrados os resultados de análise de concentrações elementares de diversas amostras de materiais de referência e de amostras de alimentos analisadas como amostras puntuais e cilíndricas. Os resultados indicam que a metodologia de análise de amostra grande ou cilíndrica pode ser aplicada na análise de amostras de alimentos.

Palavras-chave: Amostra grande, Análise por Ativação Neutrônica, Método ko padronizado, Alimentos 


\begin{abstract}
The Laboratory for Neutron Activation Analysis, Nuclear Technology Development Centre, Brazilian Commission for Nuclear Energy, CDTN/CNEN, Belo Horizonte, has developed analytical activities since the starting up of the TRIGA MARK I IPR-R1 nuclear research reactor in 1960. Nowadays, beside the relative neutron activation analysis method, the $k_{0}$-standardized method has been applied. In this method, the elemental concentration determination is not based on standards of the elements of interest, but on neutron flux monitors, absolutely calibrated gamma spectrometer, knowledge of spectral parameters in the position of irradiation of the nuclear reactor and compound nuclear constants that characterize the nuclides, so-called $\boldsymbol{k}_{0}$ factors. Over time, several improvements have been made in the analytical procedures of the laboratory, including the establishment of a methodology to analyse samples considered large, from 1 to $5 \mathrm{~g}$, when the usual neutron activation procedure is to analyse a small or punctual sample with, approximately, 200 mg. The methodology consists of analysing samples with cylindrical geometry, applying the $k_{0}$ method, without changing the local facilities. In this paper, the results of elemental concentrations of several reference materials and food samples analysed as small and large samples are shown. The results point out that the methodology of large or cylindrical sample analysis can be applied in the analysis of food samples.
\end{abstract}

Keywords: Large sample, Neutron Activation Analysis, Standardized ko method, Food 


\section{INTRODUÇÃO}

A técnica de ativação neutrônica é aplicada para a determinação da concentração multielementar em amostras diversas. O usual é analisar amostras com massas da ordem de alguns miligramas (<500 mg) ou mililitros. Esse procedimento propicia uma série de simplificações durante a irradiação, pois se considera que não há auto blindagem de nêutrons assim como não há um gradiente de fluxo de nêutrons na amostra. Durante a espectrometria gama também há simplificações, pois ao se considerar a amostra pequena ou puntual, não há auto atenuação gama [1]. Entretanto, ao se analisar uma amostra puntual, não há garantia da representatividade da amostra. Por exemplo, os elementos químicos podem não estar bem distribuídos em amostras de tecidos humanos e de alimentos [2]. Há casos em que não é permitida a retirada de uma alíquota e é preciso analisar uma quantidade maior de amostra. Por outro lado, ao se analisar uma amostra maior, há a possibilidade de se detectar maior número de elementos.

Com o objetivo de contornar essas dificuldades, uma metodologia para analisar amostras de material geológico com massas maiores do que $500 \mathrm{mg}$ até 5 gramas [3], utilizando a infraestrutura existente no CDTN, foi estabelecida. Assim, foi explorada a potencialidade da técnica de ativação neutrônica que permite que amostras com massas de gramas a quilos possam ser analisadas. Durante esse estudo, foram determinadas a auto blindagem a nêutrons e a eficiência volumétrica [4]. A metodologia estabelecida consiste em analisar amostras com forma cilíndrica, que é o formato do porta-amostra utilizado, aplicando o método $k_{0}$.

Ao longo de diversos estudos [5, 6], várias amostras de alimentos têm sido analisadas nas geometrias puntual e cilíndrica observando-se que a metodologia estabelecida pode ser aplicada também, a matrizes de alimentos, na determinação de elementos essenciais e impurezas. Neste trabalho, serão mostrados os resultados de algumas amostras que foram analisadas nos formatos puntual e cilíndrico: amostras de açúcar, de farinha de chia e de farinha branca. 


\section{MATERIAIS E MÉTODOS}

Para avaliar o desempenho do Laboratório de Ativação Neutrônica ao aplicar o método $k_{0}$, ou seja, verificar se o laboratório está produzindo resultados confiáveis, amostras de referência (NIES no 7, Tea Leaves, e NIST-SRM 1572, Citrus Leaves) foram analisadas nos formatos puntual e cilíndrico. Para isso, foi aplicado o teste estatístico de desempenho $E_{\mathrm{n}}$-score [7]. Nos cálculos, este teste considera a incerteza expandida dos valores experimentais. A avaliação do desempenho do laboratório é realizada pelo critério $\left|E_{\mathrm{n}}\right| \leq 1$, que significa que desempenho foi adequado, gerando resultados dentro do intervalo de abrangência dos valores certificados, com $95 \%$ de probabilidade de abrangência. Se $\left|E_{\mathrm{n}}\right|>1$, há a indicação de que o desempenho foi insatisfatório.

O açúcar, farinha de chia e a farinha branca foram adquiridas no comércio de Belo Horizonte. As alíquotas foram retiradas de cada amostra seguindo o procedimento usual do consumidor, sem aplicar métodos de homogeneização pois o objetivo desses estudos era verificar a presença de impurezas em alimentos [5,6]. As alíquotas foram pesadas e acondicionadas em porta amostras de polietileno adequados para a análise por ativação neutrônica. Em seguida, foram irradiadas no reator nuclear de pesquisa TRIGA MARK I IPR-R1 localizado no CDTN/CNEN, Belo Horizonte, por 8 horas, tempo suficiente para ativar os elementos com características nucleares adequadas, com secção de choque a nêutrons térmicos, meia vida, etc. Este reator, a $100 \mathrm{~kW}$, oferece fluxo médio de nêutrons térmicos de $6,4 \times 10^{11}$ nêutrons $\mathrm{cm}^{-2} \mathrm{~s}^{-1}$ na mesa giratória. Nesse dispositivo, há 40 posições de irradiação (PI), porém, as ativações foram sempre nas posições, PI-8, PI-7, PI-9. Os parâmetros espectrais $f$ (razão entre os fluxos de nêutrons térmicos e epitérmicos), igual a 22,32, e $\alpha$ (medida do afastamento da distribuição do fluxo de nêutrons epitérmicos naquela posição de irradiação em relação à distribuição ideal) igual a -0,0022, foram determinados na PI-7 e assumidos para PI-6 e PI-8. As amostras foram acompanhadas por monitores de fluxo de nêutrons (discos com 0,1mm de espessura e 6mm de diâmetro) da liga Al-Au, $(0,1 \%)$ do IRMM, Institute for Reference Materials and Measurements, Bélgica. As amostras puntuais e os monitores foram empilhados de forma intercalada em porta amostra maior. Nas amostras cilíndricas, os monitores foram colocados sob o porta-amostra contendo a amostra e na tampa deste porta-amostra.

Após irradiação, o tempo de decaimento para a amostra puntual foi de 2 a 7 dias para a determinação de elementos como o As, Br, K, La, Na, cujos radionuclídeos são de meias vidas 
médias (entre 12 e 72 horas). Para amostra cilíndrica, o tempo de decaimento foi de, no mínimo, 5 dias. Para a determinação de elementos como o $\mathrm{Cr}, \mathrm{Hg}, \mathrm{Sb}, \mathrm{Sc}, \mathrm{Zn}$, etc. cujos radionuclídeos são de meias vidas longas, o tempo de decaimento foi de 15 dias para as amostras puntuais e de, pelo menos, 20 dias para as cilíndricas.

A espectrometria gama foi executada em espectrômetro constituído por detector HPGe com $50 \%$ de eficiência nominal, calibrado previamente de forma absoluta. Para a aquisição dos espectros gama, foi utilizado o programa Genie 2k, CANBERRA. Os programas HyperLab [8] e Kayzero for Windows $^{\circledR}$ [9] foram aplicados na deconvolução dos espectros gama e na determinação da concentração elementar pelo método $k_{0}$, respectivamente.

\section{RESULTADOS E DISCUSSÃO}

Todos os cálculos de concentração elementar estão expressos com a incerteza combinada padrão ("Combined Standard Uncertainty" $[k=1]$ ), de acordo com o GUM [10]. A Tab. 1 apresenta os resultados de concentração elementar determinados para a amostra certificada NIST SRM 1572 Citrus Leaves (amostra puntual, 0,2124g; amostra cilíndrica, 2,4758g), os seus valores certificados e os calculados do $E_{\mathrm{n}}$-score. A Tab. 2 mostra os valores para a amostra de referência NIES n ${ }^{\circ}$ 7, Tea Leaves (amostra puntual, 0,3024g; amostra cilíndrica, 3,5517g).

Observa-se que todos os valores de $E_{\mathrm{n}}$-score são $\left|E_{\mathrm{n}}\right| \leq 1$, indicando que os resultados tem $95 \%$ de probabilidade de estarem dentro do intervalo de confiança. 
Tabela 1: Resultados de concentração elementar para NIST SRM 1572, Citrus Leaves.

\begin{tabular}{cccccc}
\hline & \multicolumn{5}{c}{ NIST SRM 1572 } \\
\cline { 2 - 6 } El. & $\begin{array}{c}\text { Resultado Experimental } \\
\left(\mathbf{m g ~ k g}^{-1}\right)\end{array}$ \\
& $\begin{array}{c}\text { Valor Certificado } \\
\left(\mathbf{m g ~ k g}^{-1}\right) \\
(\boldsymbol{k = 2})\end{array}$ & $\begin{array}{c}\text { Amostra } \\
\text { Puntual } \\
(\boldsymbol{k = 1})\end{array}$ & $\begin{array}{c}\boldsymbol{E}_{\mathbf{n}} \text {-score } \\
\text { Amostra } \\
\text { Cilíndrica } \\
(\boldsymbol{k}=\mathbf{1})\end{array}$ & $\boldsymbol{E}_{\mathbf{n}}$-score \\
\hline $\mathbf{A s}$ & $3,1 \pm 0,3$ & $3,5 \pm 0,1$ & 0,91 & $2,8 \pm 0,1$ & 0,78 \\
$\mathbf{B a}$ & $21 \pm 3$ & $21 \pm 2$ & 0,09 & $18 \pm 1$ & 0,97 \\
$\mathbf{C a}$ & $31500 \pm 1000$ & $34780 \pm 1554$ & 1,00 & $29150 \pm 1098$ & 0,97 \\
$\mathbf{C r}$ & $0,8 \pm 0,8$ & $0,8 \pm 0,1$ & 0,12 & $0,81 \pm 0,05$ & 0,05 \\
$\mathbf{F e}$ & $90 \pm 10$ & $109 \pm 8$ & 0,95 & $98 \pm 4$ & 0,58 \\
$\mathbf{H g}$ & $0,08 \pm 0,02$ & $<1$ & - & $0,09 \pm 0,01$ & 0,42 \\
$\mathbf{K}$ & $18200 \pm 6000$ & $20570 \pm 823$ & 0,38 & $17360 \pm 684$ & 0,14 \\
$\mathbf{N a}$ & $160 \pm 20$ & $185 \pm 7$ & 1,00 & $165 \pm 6$ & 0,22 \\
$\mathbf{R b}$ & $4,84 \pm 0,06$ & $5,3 \pm 0,5$ & 0,54 & $4,1 \pm 0,2$ & 1,75 \\
$\mathbf{S r}$ & $100 \pm 2$ & $112 \pm 8$ & 0,73 & $94 \pm 4$ & 0,61 \\
\hline
\end{tabular}

El., elemento; <, Menor do que (Limite de Detecção do elemento na amostra).

Tabela 2: Resultados de concentração elementar para NIES nº 7, Tea Leaves.

\begin{tabular}{|c|c|c|c|c|c|}
\hline \multirow{3}{*}{ El. } & \multicolumn{5}{|c|}{ NIES no 7} \\
\hline & \multirow{2}{*}{$\begin{array}{c}\text { Valor } \\
\text { Recomendado } \\
\left(\mathrm{mg} \mathrm{kg}^{-1}\right)(k=2)\end{array}$} & \multicolumn{4}{|c|}{$\begin{array}{c}\text { Resultado Experimental } \\
\left(\mathrm{mg} \mathrm{kg}^{-1}\right)\end{array}$} \\
\hline & & $\begin{array}{c}\text { Amostra } \\
\text { Puntual }(k=1)\end{array}$ & $\begin{array}{c}E_{\mathrm{n}^{-}} \\
\text {score }\end{array}$ & $\begin{array}{c}\text { Amostra } \\
\text { Cilíndrica }(k=1)\end{array}$ & $\begin{array}{c}E_{\text {n- }} \\
\text { score }\end{array}$ \\
\hline $\mathbf{B r}$ & $2,5 \pm 0,1$ & $2,8 \pm 0,1$ & 0,77 & $2,4 \pm 0,1$ & 0,56 \\
\hline $\mathbf{C a}$ & $3200 \pm 120$ & $3607 \pm 266$ & 0,75 & $2794 \pm 200$ & 0,97 \\
\hline $\mathbf{F e}$ & $98 \pm 7$ & $114 \pm 9$ & 0,84 & $98 \pm 5$ & 0,01 \\
\hline $\mathbf{K}$ & $18600 \pm 700$ & $20240 \pm 800$ & 0,94 & $18200 \pm 687$ & 0,26 \\
\hline $\mathbf{L a}$ & $0,068 \pm 0,002$ & $0,080 \pm 0,006$ & 1,00 & $0,064 \pm 0,001$ & 0,58 \\
\hline $\mathbf{N a}$ & $15,5 \pm 1,5$ & $18 \pm 1$ & 0,97 & $18 \pm 1$ & 1,00 \\
\hline $\mathbf{R b}$ & $6,59 \pm 0,01$ & $6,7 \pm 0,3$ & 0,14 & $5,7 \pm 0,5$ & 0,93 \\
\hline
\end{tabular}

El., elemento.

A Tab. 3 mostra os resultados obtidos para as amostras de alimento nas formas puntual e cilíndrica, para os elementos para os quais foram obtidos pelo menos um resultado de concentração. Para alguns elementos, os resultados de concentração ficaram abaixo do limite de detecção. Os 
resultados de concentração para os elementos que foram obtidos para os dois tamanhos de amostras, foram destacados em negrito. Alguns elementos tiveram resultados obtidos em um tipo de amostra. Para realçar, os resultados encontrados só na amostra puntual estão em itálico e os encontrados só na amostra cilíndrica, estão em itálico sublinhado.

Tabela 3: Resultado de concentração elementar das amostras de alimentos analisadas em formatos puntual e cilíndrico

\begin{tabular}{|c|c|c|c|c|c|c|}
\hline \multirow{3}{*}{ El. } & \multicolumn{6}{|c|}{ Alimentos } \\
\hline & \multicolumn{2}{|c|}{$\begin{array}{l}\text { Farinha de Chia [6] } \\
\qquad\left(\mathrm{mg} \mathrm{kg}^{-1}\right)\end{array}$} & \multicolumn{2}{|c|}{$\begin{array}{c}\text { Farinha de Trigo Branca } \\
{[6]} \\
\left(\mathrm{mg} \mathrm{kg}^{-1}\right)\end{array}$} & \multicolumn{2}{|c|}{$\begin{array}{c}\text { Açúcar Refinado [5] } \\
\left(\mathrm{mg} \mathrm{kg}^{-1}\right)\end{array}$} \\
\hline & Puntual & Cilíndrica & Puntual & Cilíndrica & Puntual & Cilíndrica \\
\hline $\mathrm{Ba}$ & $42 \pm 2$ & $36 \pm 2$ & $<3$ & $2,2 \pm 0,5$ & $<4$ & $<1$ \\
\hline $\mathrm{Br}$ & $2,19 \pm 0,08$ & $1,96 \pm 0,07$ & $1,7 \pm 0,1$ & $1,7 \pm 0,1$ & $0,020 \pm 0,002$ & $0,017 \pm 0,001$ \\
\hline $\mathrm{Ca}$ & $7059 \pm 369$ & $6120 \pm 281$ & $<500$ & $<200$ & $<200$ & $<150$ \\
\hline $\mathrm{Co}$ & $0,67 \pm 0,03$ & $\mathbf{0 , 5 7} \pm \mathbf{0 , 0 2}$ & $0,024 \pm 0,004$ & $0,015 \pm 0,005$ & $<0,01$ & $\underline{0,009 \pm 0,001}$ \\
\hline $\mathrm{Cs}$ & $0,021 \pm 0,003$ & $0,024 \pm 0,002$ & $<0,01$ & $\underline{0,012 \pm 0,001}$ & $<0,02$ & $<0,005$ \\
\hline $\mathrm{Fe}$ & $82 \pm 4$ & $100 \pm 5$ & $79 \pm 4$ & $84 \pm 4$ & $<15$ & $\underline{3,4 \pm 0,4}$ \\
\hline $\mathrm{K}$ & $7613 \pm 277$ & $6950 \pm 245$ & $1648 \pm 58$ & $1668 \pm 59$ & $8 \pm 1$ & $9 \pm 1$ \\
\hline $\mathrm{La}$ & $0,037 \pm 0,003$ & $0,033 \pm 0,002$ & $<0,03$ & $<0,004$ & $<0,01$ & $<0,002$ \\
\hline $\mathrm{Na}$ & $8,1 \pm 0,5$ & $1,8 \pm 0,1$ & $139 \pm 5$ & $135 \pm 5$ & $3,9 \pm 0,2$ & $3,8 \pm 0,1$ \\
\hline $\mathrm{Rb}$ & $8,7 \pm 0,4$ & $8,2 \pm 0,3$ & $2,0 \pm 0,1$ & $1,8 \pm 0,1$ & $<0,9$ & $<0,2$ \\
\hline $\mathrm{Sc}^{*}$ & $30 \pm 5$ & $10,4 \pm 0,4$ & $3,6 \pm 0,2$ & $3,3 \pm 0,1$ & $<1$ & $\underline{0,2 \pm 0,1}$ \\
\hline $\mathrm{Sm}$ & $0,007 \pm 0,001$ & $0,007 \pm 0,001$ & $<0,004$ & $<0,002$ & $<0,001$ & $<0,0002$ \\
\hline $\mathrm{Sr}$ & $56 \pm 3$ & $48 \pm 2$ & $<3$ & $<1$ & $<5$ & $<2$ \\
\hline $\mathrm{W}$ & $<0,02$ & $<0,01$ & $0,08 \pm 0,01$ & $<0,03$ & $<0,03$ & $<0,02$ \\
\hline
\end{tabular}

El., elemento, ${ }^{*}, \mu \mathrm{g} \mathrm{kg}^{-1} ;<$, Menor do que (Limite de Detecção do elemento na amostra).

Observando-se os resultados em negrito (ocorrência em ambas amostras) e levando em conta as incertezas dos resultados, verifica-se que os valores, na maioria deles, são compatíveis. Apenas o sódio e o escândio na amostra de farinha de chia apresentaram valores mais discrepantes ao se comparar os resultados da amostra puntual com a cilíndrica. Esse resultado sugere que a amostra de chia não estava homogênea.

Quanto as amostras de farinha de trigo e a de açúcar refinado, os valores determinados para os dois tipos de amostras, foram mais compatíveis. Além disso, em ambas foram determinados mais elementos com valores de concentração na amostra cilíndrica, evidenciando a vantagem de se analisar amostra com massa maior. 
De modo geral, os limites de detecção determinados nas amostras cilíndricas foram menores do que nas puntuais. Isso já está sendo investigado.

\section{CONCLUSÕES}

Os valores de $E_{\mathrm{n}}$-score menores do que 1, indicam o bom desempenho do Laboratório de Ativação Neutrônica ao aplicar o método $k_{0}$ tanto nas amostras puntuais como nas cilíndricas. Este critério de avaliação demonstra que o desempenho do método foi adequado, gerando resultados com 95\% de probabilidade de se encontrarem dentro da faixa de valores verdadeiros, que é o intervalo de confiança.

Os resultados das amostras de alimentos sugerem que a metodologia estabelecida para amostras geológicas, também pode ser aplicada em matriz biológica, de alimentos, e que há vantagens em se analisar amostras com massa maior.

É importante que a composição química elementar de alimentos seja conhecida ao serem determinados os elementos químicos essenciais e não essenciais. Assim, os riscos de deficiências e toxicidades de nutrientes em indivíduos e populações poderão ser avaliados.

\section{AGRADECIMENTOS}

Os autores agradecem os suportes financeiros e as bolsas às seguintes instituições: FAPEMIG (projetos Processo PPM-00143-16, APQ-01858-14; APQ-01259-09; BPV-00332-15), CNPq (APV Processo 450796/2018-3; PQ 2016, Processo 304179/2016-7), Agência Internacional de Energia Atômica (IAEA BRA 14798), CAPES e CNEN.

\section{REFERÊNCIAS}

[1] LAKMAKER, O.; BLAAUW, M. The use of big sample INAA for inhomogeneous materials: Homogenizations no longer needed? Journal of Radioanalytical and Nuclear Chemistry, v. 216, p. 69-74, 1997. 
[2] WIEL, A. V.; BLAAUW, M. Large-sample neutron activation analysis in mass balance and nutritional studies. Journal of Nutritional Science, v. 7, e. 15, p. 1-3, 2018.

[3] MENEZES, M. Â. B. C.; JACIMOVIC, R. Implementation of a methodology to analyse cylindrical 5-g sample by neutron activation technique, $k_{0}$ method, at CDTN/CNEN, Belo Horizonte, Brazil. Journal of Radioanalytical and Nuclear Chemistry, v. 300, p. 523-531, 2014.

[4] MENEZES, M. Â. B. C.; JACIMOVIC, R.; PEREIRA, C. Spatial distribution of neutron flux in geological larger sample analysis at CDTN/CNEN, Brazil. Journal of Radioanalytical and Nuclear Chemistry, v. 306, p. 611-616, 2015.

[5] SALLES, P. M. B. et al. Inorganic elements in sugar samples consumed in several countries. Journal of Radioanalytical and Nuclear Chemistry, v. 306, p. 1-9, 2015.

[6] SATHLER, M. M. Avaliação da composição elementar de alimentos integrais e refinados por meio do método $k_{0}$ de ativação neutrônica aplicado a amostras grandes. Dissertação, Programa de Pós-Graduação em Ciência e Tecnologia das Radiações, Minerais e Materiais, Centro de Desenvolvimento da Tecnologia Nuclear, 2018.

[7] INTERNATIONAL STANDARD ORGANIZATION - ISO. ISO 13528: Statistical methods for use in a proficiency testing by interlaboratory comparisons. ISO: Geneva, 2005.

[8] HYPERLAB. Gamma spectroscopy software, HyperLabs Software. Disponível em: <www.hlsof.com>. Último acesso: 18 de novembro de 2013.

[9] KAYZERO FOR WINDOWS ${ }^{\circledR}$. User's manual, for reactor neutron activation analysis (NAA) using the $\boldsymbol{k}_{\mathbf{0}}$ standardization method, Versão 2.46. $k_{0}$-ware, Heerlen, 2011.

[10] INMETRO - Instituto Nacional de Metrologia, Qualidade e Tecnologia, Avaliação de dados de medição: Guia para a expressão de incerteza de medição - GUM 2008, Duque de Caxias, RJ: INMETRO/CICMA/SEPIN, 141 p., 2012. 